\title{
Pain Management for Dental Medicine in 202I: Opioids, Coronavirus and Beyond
}

\author{
Steven J Scrivani ', David A Keith ${ }^{2}$, Ronald J Kulich $\mathbb{D}^{1,3}$, Alexandre F DaSilva ${ }^{4,5}, R$ Bruce Donoff ${ }^{2}$, \\ Shruti Handa ${ }^{2}$, Nicole Holland ${ }^{6}$, Mark A Lerman ${ }^{7}$, Jenna L McCauley ${ }^{8}$, Lori Reisner ${ }^{9,10}$, \\ Cory M Resnick ${ }^{1-13}$, Christian S Stohler ${ }^{14,15}$, Alexis Vasciannie $\mathbb{D}^{1}$, Matthew Fortino',16, \\ Michael E Schatman (iD) 1,17,18 \\ 'Department of Diagnostic Sciences, Craniofacial Pain and Headache Center, Tufts University School of Dental Medicine, Boston, MA, USA; ${ }^{2}$ Oral \\ and Maxillofacial Surgery, Harvard School of Dental Medicine, Department of Surgery, Massachusetts General Hospital, Boston, MA, USA; \\ ${ }^{3}$ Department of Anesthesia, Critical Care and Pain Medicine, Massachusetts General Hospital, Boston, MA, USA; ${ }^{4}$ Department of Biological and \\ Material Sciences \& Prosthodontics, University of Michigan School of Dentistry, Headache and Orofacial Pain Effort, University of Michigan, Ann \\ Harbor, MI, USA; ${ }^{5}$ fNIRS Laboratory, University of Michigan, Ann Harbor, MI, USA; ${ }^{6}$ Department of Public Health and Community Service, Tufts \\ University School of Dental Medicine, Boston, MA, USA; ${ }^{7}$ Department of Diagnostic Sciences, Tufts University School of Dental Medicine, \\ Boston, MA, USA; ${ }^{8}$ Department of Psychiatry and Behavioral Sciences, Medical University of South Carolina, Charleston, SC, USA; ${ }^{2}$ Department \\ of Pharmaceutical Services, School of Pharmacy University of California, San Francisco, CA, USA; ${ }^{10}$ Department of Clinical Pharmacy, San \\ Francisco Medical Center, San Francisco, CA, USA; ''Department of Plastic and Oral Surgery, Boston Children's Hospital, Boston, MA, USA; \\ ${ }^{12} \mathrm{Harvard}$ School of Dental Medicine, Boston, MA, USA; ${ }^{13} \mathrm{Harvard}$ Medical School, Boston, MA, USA; ${ }^{14}$ Columbia University College of Dental \\ Medicine, New York, NY, USA; ${ }^{15}$ Columbia University Medical Center, New York, NY, USA; ${ }^{16}$ Department of Psychiatry, Harvard Medical School, \\ Massachusetts General Hospital, Boston, MA, USA; ${ }^{17}$ Department of Public Health \& Community Medicine, Tufts University School of Medicine, \\ Boston, MA, USA; ${ }^{18}$ School of Social Work, North Carolina State University, Raleigh, NC, USA
}

Correspondence: Michael E Schatman

Email Michael.Schatman@Tufts.edu

\begin{abstract}
Over the past year our attention has inevitably been on the coronavirus pandemic, the health and welfare of our families, patients, and office staffs as well as the re-opening of our dental practices. In addition, the opioid crisis continues, is very likely to worsen as a result of the pandemic and continues to be a challenge to Dentistry. National public health issues and healthcare disparities continue and have created a global concern for providing evidence-based, adequate pain management in the dental setting. We have brought together a group of national thought leaders and experts in this field who will share their insights on the current state of opioid prescribing in Dentistry and describe some of the exciting work being done in advancing pain management.

The learning objectives for this conference proceedings were:

(1) Describing the implications of current public health concerns for safe and effective pain management in dental medicine.

(2) Identifying risk factors and understanding the current guidelines for the use of opioid and non-opioid medications in dental medicine.

(3) Analyzing the interprofessional collaborations necessary for effective pain management in dental medicine.

(4) Recognizing the challenges and opportunities brought about by the COVID-19 pandemic for the dental profession.

(5) Applying evidence-based strategies for managing the complex pain patient in the dental setting.

(6) Appraising new and future modalities for the assessment and management of orofacial pain.
\end{abstract}

Keywords: pain management, Dentistry, opioid analgesia, COVID-19 


\section{Forward}

\section{Steven J Scrivani}

Regardless of its etiology, alleviating pain is an essential right for everyone. Dental Medicine has been at the forefront of treating acute pain, whether from inflammation, infection, trauma, or pathology, in addition to post-surgical and postprocedural pain. Dentists have been well-trained to provide the proper pain management strategies for these conditions with a wide variety of modalities, including prescribing appropriate analgesic pharmacotherapy. Additionally, as the scope of Dental Medicine has evolved, there are more complex disease and patient related issues that can confound safe and effective pain management.

In 2008, the American Dental Association's Commission on Dental Accreditation (CODA) began accrediting Advanced Dental Education Training Programs in Orofacial Pain that satisfactorily met CODA Standards. One bright step occurred in March of 2020 when the National Commission on Recognition of Dental Specialties and Certifying Boards officially recognized Orofacial Pain as the 12th Dental Specialty. Currently, in the United States, there are 12 fully accredited Orofacial Pain Training Programs with a minimum of 2 years of full-time postgraduate education.

Regardless of the many advances in Dental Medicine over the past century, there continue to be numerous scientific, clinician, patient, public health, and societal issues that have affected the advancement of pain management. The rising use and abuse of opioids and opioid-related deaths plagued the profession and society for a number of years. Continuing Education specific to safe and effective pain management, risk assessment for substance use disorder, proper opioid prescribing, naloxone training, regulatory and law enforcement guidelines and follow up care are now mandatory in 24 states, and 7 states specifically define the content of these courses.

The Coronavirus pandemic has changed the way we all live and interact with one another and has certainly impacted Dental Medicine. With this, the utilization of virtual patient care visits has increased and revolutionized some aspects of dental care and will continue to impact oral healthcare in the future. Marked disparities affect all aspects of healthcare. The need for improved diagnostic criteria, more appropriate guidelines for the pediatric, adolescent, and geriatric populations, rising costs for care and the global impact of oral disorders all need to be addressed. The New Surgeon General's Report on Oral Health is expected to bring attention to these and other areas of concern.

Dental Medicine is in a position to continue as a leader in pain management and Dental Education continues to advance the concept of Interprofessional Training (Education) involving the fields of Dental Hygiene, Medicine, Nursing, Psychology, and Pharmacy. Numerous Dental Schools have adapted their curricula to meet the growing needs of contemporary healthcare in our country. The evaluation, diagnosis and management of pain is only one of these areas requiring attention.

On April 10, 2021 a Virtual Symposium was held, titled, "Pain Management for Dental Medicine: Opioids, Coronavirus and Beyond" in collaboration with Tufts University School of Dental Medicine, Harvard School of Dental Medicine, and Massachusetts General Hospital. This program, along with a Dental Clinics of North America special issue and 10 online Continuing Education modules addressing these issues were developed, tested, and disseminated through grants from Coverys Community Healthcare Foundation and RIZE Massachusetts.

This timely symposium addressed a series of issues vital to pain management in Dental Medicine for 2021 and beyond. The Proceedings in this Journal are a synopsis of the 2 Keynote addresses, and 9 presentations and discussions.

\section{Topics}

Precision Medicine for Orofacial Pain Management

Christian S Stohler, DMD, DrMedDent

The Controlled Substance Risk Assessment Program in Dentistry: Development, Barriers and Opportunities

Ronald J Kulich, PhD

Opioid Moderatism - Dental Implications

Michael E Schatman, PhD, DAAPM 
Opioid Sparing in Children and Adolescents for the Dental Provider

Cory M Resnick, DMD, MD

Non-Opioid Medications and New Pharmacotherapy in the Pipeline

Lori Reisner, PharmD, FCSHP

Telehealth in the Age of Coronavirus and Beyond

Mark A Lerman, DMD

COVID-19 Effects on the Opioid Epidemic: Lessons Learned

Jenna L McCauley, PhD

Healthcare Disparities in Oral Health and Pain Medicine: Considerations Due to the COVID-19 Pandemic

Nicole Holland, DMD

International Classification of Orofacial Pain (ICOP) - The Good, The Bad, and The Indifferent

Shruti Handa, BDS, DMD

Innovative Imaging Studies and More. What it Means for Orofacial Pain and Dental Medicine

Alexandre F DaSilva, DDS, DMedSc

Slow Ideas: Whose Time Has Come

R Bruce Donoff, DMD, MD

\section{Precision Medicine for Orofacial Pain Management} Christian S Stohler

Precision medicine, originally called personalized medicine (strongly emphasizing the role of individual gene variants) represents an emerging approach for the treatment, management and prevention of disease that acknowledges the comprehensive contribution of individual gene variants, but also environment and lifestyles. The 2011 Report of the US National Research Council, "Towards Precision Medicine: Building a Knowledge Network for Biomedical Research and a New Taxonomy of Disease" set the intellectual foundation that subsequently became a funded US initiative as identified in President Obama's 2015 State of the Union Address. ${ }^{1}$ This initiative - an extension of the Human Genome Project - should be understood as the path towards better health and health outcomes, with significant investments in infrastructure and standards comparable to the US initiative to create a national highway system. ${ }^{2}$ Clinicians will slowly depart from determining interventions based on average response rates established by means of clinical trials alone, to querying huge data lakes, representing millions of people to pair each patient with a personal matched treatment with minimized adverse reactions. This type of practice is increasingly referred to as "big data medicine", as it captures, analyzes and suggests actions using data science tools based on a wide range of genomic, molecular, cellular, physiological, clinical, behavioral, social, lifestyle and environmental parameters applicable to an individual person. In-office acquired health data will increasingly be complemented by information obtained from a host of wearable digital sensors that are interfaced with the electronic personal health record (ePHR), accessible to a growingly accountable and incentivized patient, and appropriately digested by the electronic health record owned by the respective health network system (n-EHR).

The driving factor for conceptualizing precision medicine beyond the emphasis on gene variants, acknowledging the significant contributions of risk-conferring behaviors and the environment, was based on the increasing recognition of the missing heritability of common, non-communicable diseases that represent a huge financial burden to the US healthcare system. For these health conditions - cardiovascular, metabolic, respiratory diseases, and chronic pain conditions - single genetic variants do not account for much of their heritability. ${ }^{3}$ These complex, non-communicable diseases often co-occur in the same individual, raising the question of whether the siloed, fragmented health care delivery system and existing disease taxonomies have exhausted their utility to support the best use of the accumulated science when applied to patients. The inadequacy of the US healthcare system is further put in question by established shortfalls in health outcomes, high costs for care, health inequities, well-established structural inefficiencies, missed opportunities, waste and fraud, and finally, harm to patients, calling for greater accountability, efficient learning from past mistakes and strong incentives for shaving costs but not quality of care. ${ }^{4}$ By adopting 
this new transformational thinking of precision medicine or big data medicine, the choice of care should be determined by learning from successfully treated patients that exhibit the closest "biomarker" match with the person of concern, not only informing the clinician of the best therapeutic choice for a given patient, but also avoiding harmful and costly treatment complications.

The move towards and adoption of precision medicine for orofacial pain appears to be a logical and scientifically essential choice. However, structural challenges inherent in the current care delivery system combined with professional reluctance are obvious when considering practical management issues as persistent orofacial pain conditions more likely than not - are associated with a host of comorbid ailments often beyond the scope of practice of the dental practitioner. Limitations imposed by historic scope of US State practice laws combined with the absence of fully integrated health information exchanges including medical, behavioral and dental health data by means of a shared electronic health record poses an insurmountable climb at this time. Another concern is the fact that the next generation of precision medicine-enabling taxonomies are based on fundamental knowledge of diseases assembled in network clusters that do not recognize anatomically-defined practice domains, as opposed to the current International Classification of Disease that comfortably singles out the respective specialty discipline. ${ }^{5}$ Medicaldental integration must occur for the new thinking of precision medicine/big data medicine to bear fruit in the clinical practice of orofacial pain. Nevertheless, the transition from average response medicine to applying data science in orofacial pain practice will take its time, as did the development of the national highway system. ${ }^{2}$ However, the emergence of a national highway system that ultimately yielded unprecedented interstate travel as an analogy demands a redefinition of the scientific approach to orofacial pain. Failure to do so will only hurt the discipline in the long run.

\section{References}

1. National Research Council. Towards Precision Medicine: Building a Knowledge Network for Biomedical Research and a New Taxonomy of Disease. Washington, DC: The National Academies Press; 2011.

2. Hamburg MA, Collins FS. The path to personalized medicine. N Engl J Med. 2010;363(4):301-304. doi:10.1056/NEJMp1006304

3. Manolio TA, Collins FS, Cox NJ, et al. Finding the missing heritability of complex diseases. Nature. 2009;461(7265):747-753. doi:10.1038/ nature 08494

4. Institute of Medicine. Best Care at Lower Cost: The Path to Continuously Learning Health Care in America. Washington, DC: The National Academies Press; 2013.

5. Goh K, Cusick ME, Valle D, Childs B, Vidal M, Barabás A. The human disease network. Proc Nat Acad Sci USA. 2007;104(21):8685-8690. doi:10.1073/pnas.0701361104

\section{The Controlled Substance Risk Assessment Program in Dentistry: Development, Barriers and Opportunities}

\section{Ronald J Kulich (1D)}

The development of the opioid crisis set off multiple efforts to change prescribing practices over the past 15 years, and this was paired with widespread public policy efforts to mitigate overdose risk. Healthcare providers have undergone training to better assess pain and substance use disorder risk, as well as the importance of referring their patients for mental health care. Since they largely deal with acute pain, dentists were somewhat late in recognizing these issues, and continued to unwittingly prescribe large amounts of opioids for procedures such as 3rd molar extractions. ${ }^{1}$ Nonetheless, educational outreach had an impact, and now we have seen a substantive reduction in opioid prescribing in dental practice. Indeed, there is a clear recognition that non-opioid analgesics may be equally or effective for their patients. ${ }^{2}$

Collaboration between Tufts and Harvard University faculty resulted in the award of two grants aimed at developing, validating, and dissemination a series of 10 training modules addressing Controlled Substance Risk Assessment for dentistry. These materials are now fully accessible, and we anticipate future updates.,

Although developing and providing risk mitigation content proves challenging in all areas of healthcare, there are unique barriers in dentistry. Dentists still typically fail to undertake even brief controlled substance risk assessments in their practices. This group also remains among the least adherent with respect to checking and documenting results of state Prescription Drug Monitoring Programs (PDMPs). ${ }^{5}$ There are examples of orofacial pain training programs in 
which no members of the faculty are registered with their state PDMP, while others have $100 \%$ of their staff participating in the state mandated program. We undertook an electronic dental record review for 2018 and 2019, accessing 3500 e-records. Although some subspecialty practices may have been excluded, we found only 12 e-record hits in a search using multiple PDMP terms. Are dentists failing to utilize the state PDMPs, or just failing to document the results of their searches? The answer remains unclear.

There also are few financial incentives to conduct a substance use risk assessment in dentistry. A primary care physician or nurse practitioner's practice may receive nominal financial incentives for completing formal patient risks screenings, while dental insurance typically provides no such incentives. Dental medical e-records present another major problem, as none are structured to easily include risk screeners. These records also are rarely accessible to other types of healthcare provides, a contrast to medical e-records which are increasingly assessable across multiple healthcare groups.

Despite some worthy efforts to promote interprofessional care, many dental practices are still isolated from other healthcare groups. When encountering a patient with substance use risk or a problematic PDMP result, the dentist may not have the time or enthusiasm to initiate contact with the primary care physician or mental health provider, and comfort with this effort remains lacking. Complicating the process, referral resources may be fewer for the dentist with limited access to mental health or addiction specialists. ${ }^{6}$

To better addressed these disparities across disciplines, we were afforded a novel opportunity to utilize our virtual Interprofessional Pain and Headache Rounds as a vehicle for substance use risk training, a weekly grand rounds program in operation for more than 20 years. In addition to practicing dentists and orofacial pain specialists, the Rounds attendees were broadly represented by the fields of psychology, neurology, internal medicine, addiction medicine, rehabilitation medicine, and multiple other healthcare specialties.

Utilizing this format, we provided training module access to 565 dentists over the past year, with $42 \%$ specializing in orofacial pain. Those enrolled in the complete pre-post content testing numbered 125. Our experience with the development and dissemination of this program produced a number of insights, some of which were noted above. Despite this and other national efforts to provide outreach to dentistry, barriers still exist. Public policy efforts are likely needed to change and better incentivize best-practice approaches. To our great satisfaction, we did find that subgroups of dentists reached out to us as a result of the trainings and sought to weave controlled substance risk into their practices. These self-designated "champions" typically came from the ranks of orofacial pain specialists, a group that have been on the forefront of dealing with the most complex patients who typically present with psychiatric comorbidities. We are now placing a greater focus on developing a national group of "champions," and potentially better integrating these services into the practice of dentistry.

\section{References}

1. Hoang E, Keith DA, Kulich RJ. Controlled substance misuse risk assessment and prescription monitoring database use by dentists. J Am Dent Assoc. 2019;150(5):383-392. doi:10.1016/j.adaj.2019.02.017

2. Keith DA, Kulich RJ, Bharel M, et al. Massachusetts dental schools respond to the prescription opioid crisis: a unique collaboration between the Commonwealth of Massachusetts, the Massachusetts Dental Society and the Massachusetts Dental Schools. J Dent Educ. 2017;81(12):1388-1394. doi:10.21815/JDE.017.098

3. Kulich RJ, Keith D, Schatman ME. Controlled substance risk mitigation in the dental setting. Dent Clin N Am. 2020;64(3):491-608. doi:10.1016/ S0011-8532(20)30028-8

4. Tufts University School of Dental Medicine. Controlled risk mitigation modules. Available from: https://dental.tufts.edu/continuing-education. Accessed May 6, 2021.

5. Keith DA, Shannon TA, Kulich R. The prescription monitoring program data, what it can tell you. J Am Dent Assoc. 2018;149(4):266-272. doi:10.1016/j.adaj.2018.02.015

6. Kulich RJ, Keith DA, Vasciannie A, Thomas H. Interprofessional collaboration and referral with physicians and mental health/addiction medicine specialists. Dent Clin N Am. 2020;64(3):491-608. doi:10.1016/j.cden.2020.02.002

\section{Opioid Moderatism - Dental Implications}

Michael E Schatman (iD

For roughly the past decade, the American pain care community has been in the throes of the "opioid wars", 1,2 pitting those professing "anti-opioid" sentiments against those identified as "pro-opioid". Many of the arguments made by both 
factions have been agenda-based and not particularly scientific, with considerable zealotry evident. In 2019, Schatman and colleagues ${ }^{3}$ published a call for "opioid moderatism", noting that while reverting to the clear patterns of overutilization of opioid analgesia that were evidenced during the first decade of this millennium would not be prudent, neither is the deepening climate of opiophobia and oligoanalgesia that has become progressively more prevalent in the United States over the past decade. A search for a "sane middle ground" in which opioids are prescribed judiciously as a last treatment option with prescribers responsible for exercising thorough and consistent risk mitigation was urged.

Although in 2012 dentists ranked second only to family practitioners as the leading prescribers of opioids in the US, ${ }^{4}$ their reduction in relative prescribing from $15.5 \%$ of all US opioid prescriptions to $6.4 \%$ between 1998 and 2012 was commendable. Opioid moderatism in dentistry was suggested as early as 1999, with Bullock noting the subjectivity of pain severity and the imperative of taking a phenomenological approach to each patient's analgesia needs. ${ }^{5}$ Regarding patients with chemical dependency issues, Chiodo and Tolle noted the ethical obligation of dentists to avoid simply refusing to prescribe, but rather to consider prescription within the context of multimodal care - including referral to chemical dependence specialists. ${ }^{6}$

Groundbreaking work by Denisco and colleagues ${ }^{7}$ in 2011 elucidated the importance of risk mitigation by dentists prescribing opioids, including balancing individual patients' needs for analgesia with societal responsibility, interprofessional communication, disposal of leftover opioids and drug take-back programs, use of a screening, brief intervention and referral to treatment (SBIRT) model, potential use of urine drug testing, and consultation with states' Prescription Drug Monitoring Programs (PDMPs). PDMP use among dentists remains inconsistent, with results of a 2019 national study indicating that $46.6 \%$ of dentists reported having never consulted with a PDMP, ${ }^{8}$ and a 2020 investigation determining that only $49.5 \%$ of dentists were even PDMP-registered, which was lower than physicians, nurse practitioners, physician assistants and pharmacists. ${ }^{9}$

Reducing opioid prescribing in dentistry has demonstrated its benefits, although it is important to recognize that cessation of all opioid prescribing is not going to be beneficial to many patients and to the profession. "Opioid moderatism" is heavily contingent upon prescribers taking responsibility for mitigating risk. As has been the case in non-dental medicine, failure to mitigate risk in dentistry will potentially result in opiophobia and efforts toward opioid eradication. Dentistry is already witnessing published recommendations for arbitrary prescribing limits, ${ }^{10}$ as well as methodologically flawed research "supportive" of eradicating opioid analgesia in dentistry from potentially biased investigators. ${ }^{11}$ In order to avoid causing harm to our patients and maintain acceptable levels of patient satisfaction in addition to maintaining the integrity and humanity of the profession, all dentists need to avoid becoming "anti-opioid" or "pro-opioid", and take an opioid moderatist stance of being "pro-patient". Doing so will require prescribing judiciously when opioids are necessary, as well as taking measures to mitigate risk as thoroughly and consistently as is possible.

\section{References}

1. Anderson TA. The opioid debate - CON. Clin J Pain. 2019;35(6):367-463. doi:10.1097/AJP.0000000000000702

2. Krane EJ. The opioid debate-PRO. Clin J Pain. 2019;35(6):468-472. doi:10.1097/AJP.00000000000000700

3. Schatman ME, Vasciannie A, Kulich RJ. Opioid moderatism and the imperative of rapprochement in pain medicine. J Pain Res. 2019;12:849-857. doi:10.2147/JPR.S198849

4. Gupta N, Vujicic M, Blatz A. Opioid prescribing practices from 2010 through 2015 among dentists in the United States: what do claims data tell us? J Am Dent Assoc. 2018;149(4):237-245.e6. doi:10.1016/j.adaj.2018.01.005

5. Bullock K. Dental care of patients with substance abuse. Dent Clin North Am. 1999;43(3):513-526.6.

6. Chiodo GT, Tolle SW. Ethical duties when patients make suspicious narcotic requests. Gen Dent. 2000;48(4):386-390, 392.

7. Denisco RC, Kenna GA, O'Neil MG, et al. Prevention of prescription opioid abuse: the role of the dentist. J Am Dent Assoc. 2011;142(7):800-810. doi:10.14219/jada.archive.2011.0268

8. McCauley JL, Gilbert GH, Cochran DL, et al. Prescription drug monitoring program use: national dental PBRN results. JDR Clin Trans Res. 2019;4(2):178-186. doi:10.1177/2380084418808517

9. Reist J, Frazier J, Rottingham A, Welsh M, Viyyuri BR, Witry M. Provider beliefs on the barriers and facilitators to prescription monitoring programs and mandated use. Subst Use Misuse. 2020;55(1):1-11. doi:10.1080/10826084.2019.1648512

10. Thornhill MH, Suda KJ, Durkin MJ, Lockhart PB. Is it time US dentistry ended its opioid dependence? J Am Dent Assoc. 2019;150(10):883-889. doi:10.1016/j.adaj.2019.07.003

11. Nalliah RP, Sloss KR, Kenney BC, et al. Association of opioid use with pain and satisfaction after dental extraction. JAMA Netw Open. 2020;3(3): e200901. doi:10.1001/jamanetworkopen.2020.0901 


\section{Opioid Sparing in Children and Adolescents for the Dental Provider Cory M Resnick}

The risk for future opioid abuse is increased by $33 \%$ after a single exposure to a narcotic during adolescence, even if the exposure occurs in a legitimate, prescribed fashion. ${ }^{1}$ This startling statistic emphasizes the responsibility of dental providers in the fight against opioid abuse, as the extraction of third molars is often a healthy patient's initial contact with a narcotic. ${ }^{2,3}$ Awareness, patient and family education, responsible prescribing practices, and multimodal analgesic strategies will allow significant reduction of unnecessary opiate prescriptions. ${ }^{4,5}$

Our group recently investigated the true need for opioid pain control following this common procedure. ${ }^{6}$ This was a prospective cohort study including consecutive patients presenting for extraction of four asymptomatic third molars over a 6-month period. Subjects were asked to report use of all analgesic medications for each of seven days following the procedure, and both instructions and prescriptions were standardized. Of the 81 patients who completed the study, only $6(7 \%)$ took any oxycodone over the first postoperative week; the average number of oxycodone pills used was 0.04 \pm 0.24 . Patients instead relied primarily on ibuprofen and acetaminophen, which were used for an average of $4.6 \pm 2.2$ and $3.4 \pm 1.9$ days postoperatively, respectively. Six oxycodone tablets were prescribed to each patient during the study; at the end of the study period, 466 prescribed oxycodone tablets remained unfilled or unused. The only variable that was significantly associated with a higher use of any postoperative analgesic medication was sex: females used more analgesics than males. Interestingly, factors that measured difficulty of the procedure and body mass index did not influence postoperative analgesic needs.

From our study, we concluded that the need for opioid analgesia following this common procedure is much lower than expected. Further, we found that setting expectations regarding the postoperative pain experience prior to the procedure, increasing patient and family awareness of the concern regarding unnecessary opioid prescribing, and providing a written plan to manage postoperative pain with over-the-counter medication had a significant influence on the analgesic approach that patients used. As a result of these outcomes, we decreased and eventually completely eliminated opioid prescriptions for routine third molar extraction procedures in our practice and have reinforced our peri-operative analgesia patient-family education routines. Since making these changes, we have not seen any notable increase in postoperative visits or phone calls requesting opioid analgesics.

This experience exemplifies the critical role that dental providers play in curbing opioid abuse. The third molar extraction procedure is merely an example of the many potential times that dentists may unknowingly set a child or adolescent down a path toward future opioid dependence. While patient and family education and non-narcotic analgesic strategies may encroach on the limited time available in a busy dental practice, these simple measures may significantly influence the future of our vulnerable patients.

\section{References}

1. Miech R, Johnston L, O’Malley PM, Keyes KM, Heard K. Prescription opioids in adolescence and future opioid misuse. Pediatrics. 2015;136(5): e1169-1177. doi:10.1542/peds.2015-1364

2. Maughan BC, Hersh EV, Shofer FS, et al. Unused opioid analgesics and drug disposal following outpatient dental surgery: a randomized controlled trial. Drug Alcohol Depend. 2016;168:328-334. doi:10.1016/j.drugalcdep.2016.08.016

3. Mutlu I, Abubaker AO, Laskin DM. Narcotic prescribing habits and other methods of pain control by oral and maxillofacial surgeons after impacted third molar removal. J Oral Maxillofac Surg. 2013;71(9):1500-1503. doi:10.1016/j.joms.2013.04.031

4. Denisco RC, Kenna GA, O’Neil MG, et al. Prevention of prescription opioid abuse: the role of the dentist. J AM Dent Assoc. 2011;142(7):800-810.

5. Magraw C, Pham M, Neal T, et al. A multimodal analgesic protocol may reduce opioid use after third molar surgery: a pilot study. Oral Surg Oral Med Oral Pathol Oral Radiol. 2018;126(3):214-217. doi:10.1016/j.0ooo.2018.04.007

6. Resnick CM, Calabrese CE, Afshar S, Padwa BL. Do oral and maxillofacial surgeons over-prescribe opioids after extraction of asymptomatic third molars? J Oral Maxillofac Surg. 2019;77(7):1332-1336. doi:10.1016/j.joms.2019.02.011 


\section{Non-Opioid Medications and New Pharmacotherapy in the Pipeline}

\section{Lori Reisner}

Pharmacological agents have a long history of utility in managing orofacial pain and dentists have a range of medications at their disposal, with new formulations and types becoming available especially for chronic pain conditions. Recently, COVID-19 related stresses have precipitated an increased use of opioids, alcohol and cannabinoids, ${ }^{1}$ and dental data have estimated an increased prevalence of symptomatic complaints from daytime clenching or nighttime grinding by at least $15 \%$ over prepandemic periods.

Non-steroidal anti-inflammatory drugs (NSAIDs) are effective for post-operative dental and musculoskeletal pain and are the mainstay for acute pain; they exist in several chemical subtypes ("families"), and individual patients may respond better to one family than another. NSAID use is limited by widespread toxicities including renal, gastrointestinal, cardiovascular, and central nervous system (CNS) toxicities. Relatively selective cyclooxygenase (COX-2) inhibitors such as celecoxib or meloxicam reduce GI toxicity but may carry the same or marginally greater cardiovascular risks over nonselective agents. ${ }^{3}$ Estimates attribute NSAIDs as responsible for approximately $3200-16,500$ deaths in the United States annually due to gastrointestinal hemorrhage, and current recommendations are to use the lowest effective dose of NSAIDs for the shortest time possible. ${ }^{4}$

Neuropathic pain conditions are often managed effectively with anticonvulsants or mixed-reuptake antidepressants such as amitriptyline or duloxetine. The older tricyclic antidepressants (TCAs) are considered to have greater efficacy for neuropathic pain despite having more cardiovascular and CNS toxicities than do newer agents. Both older tricyclic and newer antidepressants are contraindicated with significant heart or liver disease. Tramadol and tapentadol are options for neuropathic and non-neuropathic pains in patients who may be limited by side effects of other agents, but share potential toxicities of both antidepressants (eg, seizures) and those of opioids (eg, dizziness or confusion). Tapentadol requires caution in patients with kidney dysfunction. Gabapentin and pregabalin are generally well-tolerated with similar effectiveness to antidepressants. They are associated with drowsiness, dizziness or mood changes (eg, depression) but less often with life-threatening side effects than other drug classes for neuropathic pain.

Local anesthetics and capsaicin are also in the analgesic armamentarium when applied in stents or topically. Topical or local application significantly reduces systemic side effects. Capsaicin acceptance is limited by uncomfortable burning and need for repeated application. Multiple systematic analyses over the past 2 decades recommend antidepressants and gabapentinoids as first-line therapies (strong recommendation), followed by local anesthetics and tramadol for neuropathic pain. Opioids and botulinum are third-line recommendations.

Muscle relaxants are especially useful in temporomandibular disorders (TMD). Of these, baclofen, cyclobenzaprine and tizanidine have demonstrated intrinsic analgesic properties. Baclofen is a third-line therapy that works at the benzodiazepine receptor complex, while tizanidine is similar to clonidine and centrally reduces both pain and spasm. ${ }^{5,6}$ Cyclobenzaprine has shown utility for nighttime bruxism but is chemically related to TCAs, and thus shares many of their side effects and toxicities. Drug-drug and drug-disease interactions are always a concern when adding these to other analgesic therapies. Drugs in development demonstrating promise include many targeting sodium channels in neural membranes, derivatives of endocannabinoids, and other inhibitory compounds. ${ }^{7}$

Empirically, opioids have little proven benefit for chronic nonmalignant pain and have not demonstrated improved pain scores or functional outcomes with long-term use in most studies. Although in certain situations use of opioid medication may be warranted, ${ }^{8}$ significant safety issues dictate that practitioners optimize use of non-opioid analgesics for TMD and orofacial pain. Alternative medications include NSAIDs, local anesthetics, muscle relaxants, corticosteroids, and biological agents (eg, botulinum toxin). Mechanistically, these agents work either peripherally or centrally by decreasing a pain stimulus, pain transmission from the periphery to the central nervous system, or by altering pain perception in the cerebral cortex. Opioids reduce pain both centrally and peripherally via these pathways while antidepressants and anticonvulsants exert their effects more centrally. Genetic differences in various opioid receptor subtypes may encode for analgesic responsivity as well as risk of opioid misuse; practitioners need to be aware of both 
"high risk" (ie, likelihood of adverse events such as respiratory depression) and "high worry" (risk of aberrant behaviors) when prescribing opioids.

\section{References}

1. American Medical Association. Issue brief: reports of increases in opioid- and other drug-related overdose and other concerns during COVID pandemic; 2021. Available from: https://www.ama-assn.org/system/files/2020-12/issue-brief-increases-in-opioid-related-overdose.pdf. Accessed May 5, 2021.

2. Emodi-Perlman A, Eli I, Smardz J, et al. Temporomandibular disorders and bruxism outbreak as a possible factor of orofacial pain worsening during the COVID-19 pandemic — concomitant research in two countries. J Clin Med. 2020;9(10):3250. doi:10.3390/jcm9103250

3. Wongrakpanich S, Wongrakpanich A, Melhado K, Rangaswami J. A comprehensive review of non-steroidal anti-inflammatory drug use in the elderly. Aging Dis. 2018;9(1):143-150. doi:10.14336/AD.2017.0306

4. Cryer B. NSAID-associated deaths: the rise and fall of NSAID-associated GI mortality. Am J Gastroenterol. 2005;100(8):1694-1695. doi:10.1111/ j.1572-0241.2005.50565.x

5. Chou R, Peterson K, Helfand M. Comparative efficacy and safety of skeletal muscle relaxants for spasticity and musculoskeletal conditions: a systematic review. J Pain Symptom Manage. 2004;28(2):140-175. doi:10.1016/j.jpainsymman.2004.05.002

6. Milanov I, Georgiev D. Mechanisms of tizanidine action on spasticity. Acta Neurol Scand. 1994;89(4):274-279. doi:10.1111/j.1600-0404.1994. tb01680.x

7. Kushnarev M, Pirvulescu JP, Candido KD, Knezevic NN. Neuropathic pain: preclinical and early clinical progress with voltage-gated sodium channel blockers. Expert Opin Investig Drugs. 2020;29(3):259-271. doi:10.1080/13543784.2020.1728254

8. Khawaja SN, Scrivani SJ. Managing acute dental pain. Dent Clin North Am. 2020;64(3):525-534. doi:10.1016/j.cden.2020.02.003

\section{Telehealth in the Age of Coronavirus and Beyond}

\section{Mark A Lerman}

Depending on interpretation of the term, "telehealth" has existed for hundreds of years, with examples of telehealth service being employed during the Civil War in the form of telegraph usage. Video-based telemedicine was reported in the 1950s and 1960s and instances of teledentistry were described as early as the 1990s, yet teledentistry usage remained relatively limited until the onset of the COVID-19 pandemic. ${ }^{1,2}$ Beginning in early 2020, with many patients reluctant to seek care in person and health care practices unable to accommodate them due to capacity limits and other restrictions, telehealth has provided an opportunity both in the United States and abroad to continue providing service to patients who otherwise would have been unable to seek care. ${ }^{3}$ Early studies have suggested that, for appropriate cases, patients 4 and providers are generally satisfied with telehealth services and believe that telehealth makes it easier to receive care.

Selected departments at Tufts University School of Dental Medicine (TUSDM) began utilizing telehealth services relatively early in the pandemic. Beyond the benefit of being able to provide care to patients while clinics were closed for non-emergency services, TUSDM telehealth providers have found anecdotally an advantage of fewer canceled patient post-operative visits when compared to in-person appointments. However, teledentistry has limitations when compared to in-person visits, including intra-oral evaluation, additional documentation and the associated burden on staff, technical restrictions and the need for longer appointment times, and security concerns. Requesting submission of intra-oral photographs in advance of an appointment may supplement the patient evaluation, and this practice is recommended; however, the quality of the photographs may vary based on factors including the location of the lesion, the device used by the patient to take the photograph, and the experience of the patient as a photographer. Providers are encouraged to provide documentation in the patient's health record including: consent to undergo telehealth services; confirmation of audio and video connections; the physical location of, role of, and type of device used by each participant; and appointment start and end times. Telehealth interstate practice regulations vary by state, and while most states have modified current telehealth practice guidelines during the state of emergency, providers are encouraged to familiarize themselves with the regulations of the states in which both they and their patients are physically located. When considering which of the multiple platforms available for conducting telehealth visits to use, providers must verify that their choice of application satisfies all patient privacy concerns.

TUSDM telehealth providers have found that telehealth is not an appropriate substitute for in-person patient visits in all cases, but that some of the patients best-suited for telehealth services may be categorized as follows: new patients for whom a history may be collected remotely and/or for whom triage is necessary; established patients presenting for post-operative 
visits or follow-up of chronic conditions; patients being seen for behavioral health concerns. Even after the pandemic subsides, telehealth may persist as providers identify selected patients who may continue to benefit from virtual as opposed to in-person visits.

\section{References}

1. Glassman P, Harrington M, Namakian M, Harrison-Noonan J. The potential for telehealth technologies to facilitate charity care. Creating virtual dental homes. J Am Coll Dent. 2014;81(2):22-25.

2. Zundel KM. Telemedicine: history, applications, and impact on librarianship. Bull Med Libr Assoc. 1996;84(1):71-79.

3. Gilligan GM, Piemonte ED, Lazos JP, Panico RL. In reply to the letter to the editor "Tele(oral)medicine: a new approach during the COVID-19 crisis”. Oral Dis. 2021;27(Suppl 3):764-765. doi:10.1111/odi.13454

4. Villa A, Sankar V, Shiboski C. Tele(oral)medicine: a new approach during the COVID-19 crisis. Oral Dis. 2021;27(Suppl 3):744-745. doi:10.1111/ odi. 13364

\section{COVID-I 9 Effects on the Opioid Epidemic: Lessons Learned} Jenna L McCauley

Prior to the COVID-19 worldwide pandemic and associated mitigation measures, the United States had entered the "fourth-wave" of an opioid epidemic. Whereas earlier waves of the epidemic were characterized by increases in overdose deaths primarily attributable to prescription opioids (first wave), heroin (second wave), and synthetic illicit opioids (third wave), the country was beginning to experience upticks in overdose deaths involving psychostimulants such as methamphetamine and cocaine. ${ }^{1}$ However, multi-level interventions addressing licit and illicit drug supplies, prevention, treatment expansion, and naloxone distribution culminated in 2018, contributing to the first national decline in drug overdose deaths in 28 years. ${ }^{2}$ Two cornerstones of these nationwide efforts to decrease risk for overdose included expansion of access to: (1) gold-standard medication for opioid use disorder (MOUD), including methadone, buprenorphine, and naltrexone; and (2) the overdose reversing agent, naloxone.

Systemic disparities in access to treatment, as well as financial instability and associated gaps in insurance coverage, were key drivers of the opioid epidemic prior to the pandemic. ${ }^{4,5}$ Early data regarding the impacts of COVID-19 and associated mitigation measures suggest that these disparities have been exacerbated, likely contributing to upticks in fatal and non-fatal overdose specifically, and increased substance abuse behaviors more broadly. ${ }^{6-8}$

Strong evidence supports the effectiveness of MOUD in reducing all-cause and overdose related mortality and suggests that substance use treatment response falls in line with that of other chronic, relapsing conditions such as hypertension, type 2 diabetes, and asthma. ${ }^{9,10}$ In spite of these realities, treatment for substance use disorders, including MOUD, has continued to be largely siloed from mainstream healthcare, perhaps making it particularly vulnerable to pandemic-related impacts. ${ }^{11}$ Though delays in care and the impacts of diminished healthcare expenditures had a widespread impact across the healthcare sector, ${ }^{12}$ these impacts appeared to more severely affect services that were less amenable - for a variety of reasons - to virtual care delivery. ${ }^{13}$ Similar to dental medicine, addiction medicine was particularly hard hit by both individual/patient-level delays in seeking care, as well as systemic closure or limited-service provision by substance use treatment providers as a result of the COVID-19 pandemic and associated mitigation measures.

In response to the widespread effects of the COVID-19 pandemic, several temporary but noteworthy national measures were put into place to address disruptions in MOUD access and naloxone availability by increasing the provider base and decreasing barriers to access. Measures broadly included increased flexibility for MOUD providers, such as: allowing take-home dosing for stable methadone patients, waiving the requirement for an in-person initial visit prior to initiating treatment with buprenorphine via telehealth, and encouragement of waivers of counseling and psychosocial treatment requirements when these presented as a barrier to patients receiving MOUD. These measures, though currently temporary, present a unique opportunity to evaluate the impact of these policy-level changes on MOUD treatment access, utilization, and outcomes, as well as on associated safety data regarding diversion and adverse events. ${ }^{14}$ Planned investigations are currently in their early stages. ${ }^{15}$ 
Despite the deserved attention paid to the increasing role of illicit drugs (particularly fentanyl and psychostimulants) in the overdose epidemic, as well as noted national declines in both opioid prescribing and prescription opioid-involved overdose, it is important to note that prescription opioids continue to contribute to a substantial number of overdose deaths each year. ${ }^{1}$ Although more recent data are needed, data predating the COVID-19 pandemic suggest that patterns of opioid prescribing vary by location and that a minority of dentists continue to prescribe opioids in excess of recommendations for acute pain management. ${ }^{16,17}$ Continued vigilance among dental prescribers is warranted when considering opioids for pain management, particularly among adolescents and young adults, given the potential risk conferred for future opioid use and abuse. ${ }^{18}$ Consistent with American Dental Association recommendations, ${ }^{19}$ dental prescribers should standardly consider non-opioid alternatives as first-line pain management options, and if prescribing an opioid, should utilize prescription drug monitoring resources and provide thorough patient counseling regarding pain management expectations and opioid risk mitigation strategies (eg, secure storage, timely disposal).

\section{References}

1. Centers for Disease Control and Prevention (CDC). Multiple cause of death 1999-2019 on CDC wonder online database. National Center for Health Statistics; December, 2020. Available from: https://wonder.cdc.gov/mcd.html. Accessed April 3, 2021.

2. Centers for Disease Control and Prevention (CDC). Life expectancy increases in 2018 as overdose deaths decline along with several leading causes of death. National Center for Health Statistics Data Brief No. 355; January, 2020. Available from: https://www.cdc.gov/nchs/products/databriefs/ db355.htm. Accessed April 3, 2021.

3. Madras BK. The President's Commission on combating drug addiction and the opioid crisis: origins and recommendations. Clin Pharmacol Ther. 2018;103(6):943-945. doi:10.1002/cpt.1050

4. Goedel WC, Shapiro A, Cerdá M, Tsai JW, Hadland SE, Marshall BDL. Association of racial/ethnic segregation with treatment capacity for opioid use disorder in counties in the United States. JAMA Netw Open. 2020;3(4):e203711. doi:10.1001/jamanetworkopen.2020.3711

5. Orgera K, Tolbert J The opioid epidemic and Medicaid's role in facilitating access to treatment. Kaiser Family Foundation Issue Brief; May, 2019. Available from: https://files.kff.org/attachment/Issue-Brief-The-Opioid-Epidemic-and-Medicaids-Role-in-Facilitating-Access-to-Treatment. Accessed April 3, 2021.

6. Enns A, Pinto A, Venugopal J, et al. Substance use and related harms in the context of COVID-19: a conceptual model. Health Promot Chronic Dis Prev Can. 2020;40(11-12):342-349. doi:10.24095/hpcdp.40.11/12.03

7. Holland KM, Jones C, Vivolo-Kantor AM, et al. Trends in US emergency department visits for mental health, overdose, and violence outcomes before and during the COVID-19 pandemic. JAMA Psychiatry. 2021;78(4):372-379. doi:10.1001/jamapsychiatry.2020.4402

8. Pollard MS, Tucker JS, Green HD Jr. Changes in adult alcohol use and consequences during the COVID-19 pandemic in the US. JAMA Netw Open. 2020;3(9):e2022942. doi:10.1001/jamanetworkopen.2020.22942

9. Sordo L, Barrio G, Bravo MJ, et al. Mortality risk during and after opioid substitution treatment: systematic review and meta-analysis of cohort studies. BMJ. 2017;357:j1550. doi:10.1136/bmj.j1550

10. McLellan AT, Lewis DC, O'Brien CP, Kleber HD. Drug dependence, a chronic medical illness: implications for treatment, insurance, and outcomes evaluation. JAMA. 2000;284(13):1689-1695. doi:10.1001/jama.284.13.1689

11. McCauley JL, McLellan AT. Treating addiction treatment like a chronic illness: a practical clinical model. In: Brady KT, Levin F, Galanter M, Kleber HD, editors. The American Psychiatric Press Textbook of Substance Abuse Treatment, Sixth Edition. American Psychiatric Association Publishing; 2021.

12. Robert Wood Johnson Foundation (RWJF). The impact of coronavirus on households across America; September, 2020. Available from: https:// www.rwj.org/en/library/research/2020/09/the-impact-of-coronavirus-on-households-across-america.html\#: :text=Across\%20rural\%20American\% 2C\%20more $\% 20$ than,hours $\% 20$ during $\% 20$ the $\% 20$ coronavirus $\% 20$ outbreak.\&text $=$ One $\% 20$ in $\% 20$ five $\% 20$ households $\% 20$ (20,problems $\% 20$ during \%20the\%20coronavirus\%20outbreak. Accessed April 3, 2021.

13. Health Affairs Blog. COVID-19 shocks the US Health Sector: a review of early economic impacts; December 16, 2020. Available from: https:// www.healthaffairs.org/do/10.1377/hblog20201214.543463/full/. Accessed April 3, 2021.

14. Nunes EV, Levin FR, Reilly MP, El-Bassel N. Medication treatment for opioid use disorder in the age of COVID-19: can new regulations modify the opioid cascade? J Subst Abuse Treat. 2021;122:108196. doi:10.1016/j.jsat.2020.108196

15. Livingston NA, Ameral V, Banducci AN, Weisberg RB. Unprecedented need and recommendations for harnessing data to guide future policy and practice for opioid use disorder treatment following COVID-19. J Subst Abuse Treat. 2021;122:108222. doi:10.1016/j.jsat.2020.108222

16. Dana R, Azarpazhooh A, Laghapour N, Suda KJ, Okunseri C. Role of dentists in prescribing opioid analgesics and antibiotics: an overview. Dent Clin North Am. 2018;62(2):279-294. doi:10.1016/j.cden.2017.11.007

17. Suda KJ, Zhou J, Rowan SA, et al. Overprescribing of opioids to adults by dentists in the U.S., 2011-2015. Am J Prev Med. 2020;58(4):473-486. doi:10.1016/j.amepre.2019.11.006

18. Schroeder AR, Dehghan M, Newman TB, Bentley JP, Park KT. Association of opioid prescriptions from dental clinicians for US adolescents and young adults with subsequent opioid use and abuse. JAMA Intern Med. 2019;179(2):145-152. Erratum in: JAMA Intern Med. 2019;179(7):1007. doi:10.1001/jamainternmed.2018.5419

19. American Dental Association (ADA). Statement on the use of opioids in the treatment of dental pain; October, 2016. Available from: https://www. ada.org/en/advocacy/current-policies. Accessed April 3, 2021. 


\title{
Healthcare Disparities in Oral Health and Pain Medicine: Considerations Due to the COVID-19 Pandemic
}

\author{
Nicole Holland
}

Disparities in both fields of oral health and pain medicine are well-documented. Although the topic of healthcare disparities is not new, COVID-19 has further demonstrated the vast inequities within our healthcare system. Healthcare disparities refer to differences in medical service access and utilization, as well as variations in disease rates between population groups across various socioeconomic characteristics. ${ }^{1}$ Social determinants of health - conditions in the places where people live, learn, work, and play that affect a wide range of health risks and outcomes ${ }^{2}$ - not only contribute to healthcare disparities but remain imperative issues for pain medicine and oral health practitioners, especially during the pandemic and as society recovers from its devastating effects.

Certain racial and socioeconomic groups have poorer oral health; one example is that non-Hispanic Black or Mexican-American adults display twice the rates of untreated cavities as do non-Hispanic whites. ${ }^{3}$ Additionally, lowincome or uninsured adults are twice as likely to have 1-3 untreated cavities and 3 times more likely to have 4 or more untreated cavities as do adults with higher income levels or dental insurance. ${ }^{3,4}$ Parallel statistics can be found in the field of pain medicine as well, with Blacks less likely than Whites to receive analgesic medication for pain, regardless of age or socioeconomic status. ${ }^{4,5}$

Although the US experience with the coronavirus pandemic is just over a year old, COVID-19 has both further exacerbated the aforementioned healthcare inequities and magnified the social and economic factors that contribute to them. Not only have racial and ethnic minorities experienced disproportionately higher rates of COVID infection, hospitalization, and deaths, ${ }^{6}$ but the pandemic has also produced disparate effects on socioeconomic conditions in communities of color. One example has been employment, with minorities experiencing higher rates of job loss. In April 2020, national unemployment rates for Blacks and Hispanics were $16.7 \%$ and $18.9 \%$, respectively, compared to $14.2 \%$ for Whites. ${ }^{7}$ Employment directly impacts financial means, food security, housing, insurance, and downstream outcomes such as dental care and pain medicine utilization.

Unfortunately, not only was access to health services drastically reduced when the pandemic started without regard to existing needs, but government agencies and professional organizations at the highest levels provided inconsistent information regarding health information and guidelines. Consider the timeline of oral health recommendations during the early stages of the pandemic. The World Health Organization (WHO) officially declared the coronavirus outbreak a pandemic on March 11, 2020. ${ }^{8}$ On March 16, the American Dental Association (ADA) recommended that dental practices limit their services to only emergency care until April $6,{ }^{9}$ which was later extended to April 30. The CDC also recommended postponing all non-essential dental services. ${ }^{10}$ It was not until August 3 that the WHO issued interim guidance advising the delay of all non-urgent dental care until sufficient reduction in community COVID-19 transmission rates were achieved, nearly 5 months after its first pandemic declaration. ${ }^{11}$ To make matters more confusing, the ADA then responded with a public statement that "respectfully and strongly" disagreed with said WHO guidelines from August 3. ${ }^{12}$ Research has shown that clear, effective health communication informs and influences health decisions. During COVID-19, not only were healthcare services limited but health information was also disseminated in ways that were not equally understandable to different groups. ${ }^{13}$ From the provider side, clinics forced to limit their services for several months also dealt with inconsistent reopening criteria across geographic areas, while also facing financial pressures and social distancing requirements. From the patient side, this likely exacerbated already-existing healthcare disparities in oral health and pain medicine for vulnerable populations. Even though the full extent of pandemic-related data are not yet available, alreadyvulnerable populations likely had greater difficulty with access and care utilization during the pandemic than did other already-healthier individuals.

Post-pandemic recovery efforts must consider healthcare disparities as a key issue. As such, COVID-19 threatens to increase disparities in both oral health and pain medicine at all levels. Health providers and institutions must take this 
into account at individual- and population-health levels so that future policies and programs may systematically tackle both existing and newly derived healthcare disparities.

\section{References}

1. Agency for Healthcare Research and Quality. Disparities. Available from: https://www.ahrq.gov/topics/disparities.html. Accessed March $29,2021$.

2. Centers for Disease Control and Prevention. About social determinants of health. Available from: https://www.cdc.gov/socialdeterminants/about. html. Accessed April 6, 2021.

3. Centers for Disease Control and Prevention. Oral health surveillance report: trends in dental caries and sealants, tooth retention, and edentulism, United States, 1999-2004 to 2011-2016. US Dept of Health and Human Services; 2019. Available from: https://www.cdc.gov/oralhealth/ publications/OHSR-2019-index.html. Accessed April 1, 2021.

4. Institute of Medicine (IOM). Relieving Pain in America: A Blueprint for Transforming Prevention, Care, Education, and Research. Washington, DC: The National Academies Press; 2011.

5. Anderson KO, Green CR, Payne R. Racial and ethnic disparities in pain: causes and consequences of unequal care. $J$ Pain. 2009;10 (12):1187-1204. doi:10.1016/j.jpain.2009.10.002

6. Artiga S, Garfield R, Orgera K Communities of color at higher risk for health and economic challenges due to COVID-19. KFF; April 7, 2020. Available from: https://www.kff.org/coronavirus-covid-19/issue-brief/communities-of-color-at-higher-risk-for-health-and-economic-challenges-due -to-covid-19/. Accessed April 8, 2021.

7. US Bureau of Labor Statistics. Unemployment rate rises to record high 14.7 percent in April 2020; 2020. Available from: https://www.bls.gov/ opub/ted/2020/unemployment-rate-rises-to-record-high-14-point-7-percent-in-april-2020.htm. Accessed April 8, 2021.

8. World Health Organization. Listings of WHO's response to COVID-19; 2020. Available from: https://www.who.int/news/item/29-06-2020covidtimeline. Accessed April 8, 2021.

9. American Dental Association. ADA News - ADA recommending dentists postpone elective procedures; 2020. Available from: https://www.ada. org/en/publications/ada-news/2020-archive/march/ada-recommending-dentists-postpone-elective-procedures. Accessed April 7, 2021.

10. Centers for Disease Control and Prevention. Framework for healthcare systems providing non-COVID-19 clinical care during the COVID-19 pandemic; 2020. Available from: https://www.cdc.gov/coronavirus/2019-ncov/hcp/framework-non-COVID-care.html. Accessed April 7, 2021.

11. World Health Organization. Considerations for the provision of essential oral health services in the context of COVID-19: interim guidance; 2020. Available from: https://www.who.int/publications/i/item/who-2019-nCoV-oral-health-2020.1. Accessed April 7, 2021.

12. American Dental Association. American Dental Association responds to World Health Organization recommendation: dentistry is essential healthcare; 2020. Available from: https:/www.ada.org/en/press-room/news-releases/2020-archives/august/american-dental-association-dentistry-isessential-health-care?utm_source=adaorg\&utm_medium=alertbar\&utm_content=response-WHO\&utm_campaign=covid-19. Accessed April 7, 2021.

13. Caballero A, Leath K, Watson J. COVID-19 consumer health information needs improvement to be readable and actionable by high-risk populations. Front Commun. 2020;5:56. doi:10.3389/fcomm.2020.00056

\section{International Classification of Orofacial Pain (ICOP)- The Good, The Bad, The Indifferent}

\section{Shruti Handa}

Orofacial Pain [OFP] can affect $7-11 \%$ of the population. The anatomic complexity of the region and lack of information on the pathophysiology and treatment make these conditions challenging to diagnose and hence manage. ${ }^{1}$ More often they end up falling under the diagnosis of "Atypical Facial Pain"(AFP).

For many years, clinicians and the orofacial pain community have relied on the classification systems established by the International Headache Society [IHS], International Association for the Study of Pain [IASP], American Academy of Orofacial Pain [AAOP] and the Diagnostic Criteria for Temporomandibular Disorders [DC-TMD]. However, potential weaknesses in the application of these classification systems to the diverse OFP population have been recognized and addressed in several studies. ${ }^{2}$ Benoliel and colleagues reported a diagnostic efficiency of $56 \%$ when employing IHS criteria alone in diagnosing OFP patients. A successful diagnosis of $92.7 \%$ required implementation of three classification systemsIHS, AAOP, DC-TMD. ${ }^{3}$ Therefore, a consensus among the medical and dental community attempted to establish a comprehensive classification system for orofacial pain diagnosis.

A collaborative group consisting of members of the AAOP, IHS, Orofacial and Head Pain Special Interest Group (OFHP SIG) of the IASP, and the International Network for Orofacial Pain and Related Disorders Methodology (INFORM) compiled the first edition of the International Classification of Orofacial Pain [ICOP-1st edition], which was published in 2020. The ICOP is aligned with the International Classification of Diseases - 11th revision [ICD-11] and IASP criteria for orofacial pain and headaches. ${ }^{4}$ It provides a comprehensive description and diagnostic criteria for orofacial pain conditions, which are classified under 6 main categories, to include pain in the dentoalveolar and 
anatomically related structures, myofascial orofacial pain, temporomandibular joint pain, orofacial pain attributed to lesions or disease of the cranial nerves, pain resembling primary headaches, and idiopathic orofacial pain. The classification system aids in facilitating the diagnosis of complex orofacial pain conditions. It is beneficial for all clinicians and especially for dentists, who are among the first healthcare providers to be consulted by orofacial pain patients, followed by neurologists and neurosurgeons.

Although dental pain is the most common orofacial pain condition seen by dentists, other difficult orofacial pain diagnoses that dentists and primary care physicians can be confronted with include, but are not limited to, myofascial orofacial pain, temporomandibular joint [TMJ] pain, trigeminal neuralgia [TN], post traumatic trigeminal neuropathic pain [PTTN], burning mouth syndrome [BMS], persistent idiopathic facial pain [PIFP] and persistent idiopathic dentoalveolar pain [PIDP]. Myofascial orofacial pain is quite prevalent in the adult population, can refer to the teeth and odontogenic structures, and mimic a toothache. Likewise, the pain of TN, PTTN, PIFP and PIDP can masquerade as odontogenic pain. The pain in BMS is more distinct and is mostly burning in quality, with the tongue being the most common site. It has been estimated that almost 53\% patients with non-odontogenic orofacial pain, especially trigeminal neuralgia, are treated/ mistreated for odontogenic pain. ${ }^{5}$ Therefore, understanding the differential diagnosis of OFP is imperative for dentists.

ICOP facilitates the diagnosis of these conditions which were previously called AFP. The diagnostic criteria will increase understanding and improve safe and effective management of OFP patients.

\section{References}

1. Zakrzewska JM. Multi-dimensionality of chronic pain of the oral cavity and face. J Headache Pain. 2013;14(1). doi:10.1186/1129-2377-14-37

2. Benoliel R, Eliav E, Sharav Y. Classification of chronic OFP: applicability of chronic headache criteria. Oral Surg Oral Med Oral Pathol Oral Radiol Endod. 2010;110(6):729-737. doi:10.1016/j.tripleo.2010.07.009

3. Benoliel R, Birman N, Eliav E, Sharav Y. The International Classification of Headache Disorders: accurate diagnosis of orofacial pain? Cephalalgia. 2008;28(7):752-762. doi:10.1111/j.1468-2982.2008.01586.x

4. Orofacial T, Classification P. International Classification of Orofacial Pain, 1st edition (ICOP). Cephalalgia. 2020;40(2):129-221.

5. Von Eckardstein KL, Keil M, Rohde V. Unnecessary dental procedures as a consequence of trigeminal neuralgia. Neurosurg Rev. 2015;38 (2):355-360. doi:10.1007/s10143-014-0591-1

\section{Innovative Imaging Studies and More. What It Means for Orofacial Pain and Dental Medicine} Alexandre F DaSilva

Pain is the most common reason for seeking medical care, and in many patients, it severely impairs their daily lives. Unfortunately, existing methods for assessing and tracking an individual's pain are mainly based on a self-report visual analogue scale ranging from 0 to 10 (VAS: from 0 [no pain] to 10 [worst pain]) and are entirely subjective - making it difficult to interpret data or ascertain the level of precision necessary before, during and after experiencing pain and between visits, trials or procedures to support evidence-based pain management practices. For many years clinicians have been seeking precise pain assessment solutions via neuroimaging techniques, focusing on the brain for a human pain biomarker. Unfortunately, most of those techniques are not applicable in the clinical environment or lack accuracy.

Recent studies have demonstrated that interactive mobile and 3D visualization technologies can optimize training, planning, and efficiency in healthcare, bringing more accurate information to doctors, patients, and students. Starting with 3D-mobile technologies, they have advanced the goal to create a "Pain-GPS" to optimize monitoring and analysis of pain intensity and localization, at individual and populational levels, across diverse patients' body types (eg, age, gender). ${ }^{1}$ However, they are still based on the patients' reports. Although not yet perfected, the combination of neuroimaging, artificial intelligence, computational power, and computer vision techniques opens a window for the newera pain measurement. Researchers have analyzed the neurological signature of pain using functional magnetic resonance imaging (fMRI), and, more recently, positron emission tomography to measure molecular mu-opioid and dopamine dysfunction in vivo during chronic TMD and migraine attacks. ${ }^{2-4}$ Machine learning technology on data collected with neuroimaging has begun to illustrate the possibility of detecting a robust neurological signature of pain at the person's 
level using those tools. Other researchers have demonstrated the possibility of detecting even TMD using multi-voxel pattern analysis on fMRI signal. This new neuroimaging gold rush to report brain-pain biomarkers prompted the field to recommend standards of evidence. ${ }^{5}$ Indeed, fMRI-based objective assessments of pain have provided a significant step ahead in the path of dissecting the brain mechanisms of pain, yet the size and cost of the MRI scanner and other neuroimaging tools (eg, PET, magnetoencephalography) prevent their application in the clinical setting.

The size and cost limitations in neuroimaging have sparked the interest in portable devices with similar technical benefits as fMRI. Functional near-infrared spectroscopy (fNIRS) detects concentration variations of oxy-hemoglobin $(\mathrm{HbO})$ and deoxy-hemoglobin $(\mathrm{Hb})$, such as blood oxygen level-dependent (BOLD) signal in fMRI. It measures the absorption of near-infrared light at wavelengths between 700 and $1000 \mathrm{~nm}$, non-invasively through the skull. ${ }^{6,7}$ fNIRS is a portable neuroimaging device with similar technical benefits as fMRI. Recent fNIRS studies have concentrated on accessible key cortical regions for pain processing, facilitating neuro-engineering design (eg, sensor numbers and placement) with maximum output and benefit for pain decoding. For example, it is now possible with fNIRS to evaluate a patient's cortical activation before, during, and after dental hypersensitivity pain with the patient in the dental chair. ${ }^{89}$ Researchers reported well-defined hemodynamic cortical activations in two regions, primary sensory (S1) and prefrontal cortices (PFC), which were elicited by thermal stimulation to the affected tooth from expectation to pain detection. The patient's clinical pain experience was fully predicted concomitantly by the baseline functional connectivity between S1 and PFC and a well-defined stepwise sequence of hemodynamic cortical responses. ${ }^{9}$ The sensory-discriminative and cognitive-emotional cascade of brain responses were initiated during the clinical pain expectation (pre-pain phase) with activations in the contralateral S1 orofacial homuncular region and also in the bilateral PFC. Such activations were followed by flat response or deactivation at PFC and further S1 responses when the cold stimuli crossed noxious levels (pain phase). Following such findings, the group developed a framework technology that objectively visualizes, measures, and decodes in real-time the ongoing cascade of cortical activities when and where there is clinical pain to optimize pain diagnosis, treatment and training. This framework was successfully tested in patients experiencing dental hypersensitivity with evoked pain through 3-step experiments integrating clinical (optical) neuroimaging, augmented reality (AR), and neural network-based artificial intelligence (AI), called CLARAi. ${ }^{10}$ Additional studies are needed to expand brain-pain biomarkers to other conditions and contexts in the clinical environment, such as in high-risk decisionmaking processes in dental and medical emergency settings. The concept of "seeing pain" directly from the patient's brain in the clinic is achievable. ${ }^{11}$

\section{References}

1. Kaciroti N, DosSantos MF, Moura B, et al. Sensory-discriminative 3D-body pain mobile app measures prevail over moody VAS: a validation study. JMIR Mhealth Uhealth. 2020;8(8):e17754. doi:10.2196/17754

2. Jassar H, Nascimento TD, Kaciroti N, et al. Impact of chronic migraine attacks and their severity on the endogenous mu-opioid neurotransmission in the limbic system. Neuroimage Clin. 2019;23:101905. doi:10.1016/j.nicl.2019.101905

3. Nascimento TD, Yang N, Salman D, et al. Micro-opioid activity in chronic TMD pain is associated with COMT polymorphism. $J$ Dent Res. 2019;98(12):1324-1331. doi:10.1177/0022034519871938

4. Kim DJ, Jassar H, Lim M, Nascimento TD, DaSilva AF. Dopaminergic regulation of reward system connectivity underpins pain and emotional suffering in migraine. $J$ Pain Res. 2021;14:631-643. doi:10.2147/JPR.S296540

5. Davis KD, Flor H, Greely HT, et al. Brain imaging tests for chronic pain: medical, legal and ethical issues and recommendations. Nat Rev Neurol. 2017;13(10):624-638. doi:10.1038/nrneurol.2017.122

6. Ferrari M, Quaresima V. A brief review on the history of human functional near-infrared spectroscopy (fNIRS) development and fields of application. Neuroimage. 2012;63(2):921-935. doi:10.1016/j.neuroimage.2012.03.049

7. Obrig H, Villringer A. Beyond the visible-imaging the human brain with light. J Cereb Blood Flow Metab. 2003;23(1):1-18. doi:10.1097/01. WCB.0000043472.45775.29

8. Racek AJ, Hu X, Nascimento TD, et al. Different brain responses to pain and Iits expectation in the dental chair. J Dent Res. 2015;94(7):998-1003. doi:10.1177/0022034515581642

9. Hu X, Racek AJ, Bellile E, et al. Brain functional changes before, during, and after clinical pain. J Dent Res. 2018;97(5):523-529. doi:10.1177/ 0022034517750136

10. Hu XS, Nascimento TD, Bender MC, et al. Feasibility of a real-tme clinical augmented reality and artificial intelligence framework for pain detection and localization from the brain. $J$ Med Internet Res. 2019;21(6):e13594. doi:10.2196/13594

11. Hu X-S, Nascimento TD, DaSilva AF. Shedding light on pain for the clinic: a comprehensive review of using functional near-infrared spectroscopy to monitor its process in the brain. Pain. 2021. 


\section{Slow Ideas: Whose Time Has Come}

\section{R Bruce Donoff}

Previously, we have suggested that change in healthcare delivery must include oral health. Recent interest in this issue is based upon several studies strongly suggesting that inclusion of oral health in primary care improves access to care and health outcomes while reducing overall healthcare costs. ${ }^{1,2}$ Multiple solutions have been offered for this integration, ranging from interprofessional education to innovative degree programs. The issue is how to break down the silos that have developed between medicine and dentistry. As Casciaro et al suggest ${ }^{3}$ this requires either redesign of formal organizational structures, which can be costly and slow, or identification and implementation of activities that facilitate boundary crossing and cultural change. Ellner and Phillips in their analysis ${ }^{4}$ suggest that the principles of team care will require new approaches to workforce training and education.

In addition, the dental profession could change its scope by providing greater general health evaluation and advice. As examples, consideration of sleep apnea by dentists already exists. The COVID-19 pandemic is bringing out the best in our providers, many of whom are providing vaccinations to patients. In addition to developing teams that could provide HPV vaccinations as part of a comprehensive campaign against oral cancer, dentists can use team approaches to positively impact opioid and other substance abuse. The culture must change and enable dentists to consider themselves and be perceived by medical peers and patients as part of the team providing "whole person care." Multiple reports, from the 1995 Institute of Medicine study, ${ }^{5}$ the first-ever Surgeon General's Report ${ }^{6}$ in 2001, to the recent "Advancing Dental Education in the 21 st Century" report ${ }^{7}$ have raised critical issues that impede oral health integration but offer little guidance for change. The last report, building on the landmark Gies Report of $1926,{ }^{8}$ dentistry's Flexner Report equivalent, started with the premise that dentistry must be like ophthalmology (part of the whole person care team) and not optometry (technical service providers) but fell short on recommendations for change that could address the cultural divide and promote integration. The data suggest an overall decrease in science and whole patient health education among dental schools, persistence of procedural training, and lack of mandatory internships, underscoring the marginalization of dentists from the rest of the health care team. Opportunities for building interprofessional education exist, like medical-dental school alliances, but cross appointment of faculty is rare, as is recruitment of dual-degree faculty who have the breadth of training and experience to teach in both schools. Professional education in healthcare is frequently dominated by values of independence and autonomy, not collaboration.

There is a critical need for leaders who see integrative care as being of paramount importance and who can advocate for oral health inclusion in general health care, from prevention to chronic disease management to tertiary care. Currently, there are few individuals who can advocate effectively within government departments or other high-level policy-making organizations. Leaders must combine intimate knowledge of current dental care delivery and reimbursement with public health skills to accomplish successful integration of oral health with evolving payment approaches. ${ }^{9}$ To give such individuals clout they would require first-hand knowledge of oral health through unique educational and training programs. A general practice residency within a department of medicine with new parameters would equip individuals with a sophisticated understanding of the medical and dental healthcare systems and clinical practice. These leaders could enhance the public's health both by leading model interprofessional teaching practices which include medical, dental, nursing, hygiene, and pharmacy students and by championing oral health in policy debates. The opportunity to unify dental and medical education was missed over a century ago and the goal now should be creation of multidisciplinary teams in interprofessional teaching practices, led by appropriately prepared leaders who can lead by crossing professional boundaries without major changes in state regulatory rules.

Louis Menand offers the following insight: the key to reform in higher education lies not in the way that knowledge is produced. ${ }^{10}$ It lies in the way that the producers of knowledge are produced. I would extend this to include professional education in dental and medical schools. We are not bound to live by the decisions that kept the Baltimore College of Dentistry separate in 1840. Transformation is possible. What will the legacy of the COVID-19 pandemic be for improvements in health care in general and oral health in particular? Relevant to the topic of this conference, how could the creation of interdisciplinary and interprofessional teams enhance efforts to reduce opioid misuse? 
As the use of interdisciplinary and interprofessional teams has been empirically associated with safer opioid prescribing in general medicine, ${ }^{11}$ there is no reason to believe that such an approach cannot be directly relevant to dental medicine, as well.

\section{References}

1. Murthy VH. Oral health in America 2000 to present: progress made, but challenges remain. Public Health Rep. 2016;131(2):224-225. doi:10.1177/ 003335491613100202

2. Hummel J, Phillips KE, Holt B, Hayes C. Oral health: an essential component of primary care. Qualishealth.org; June, 2015. Available from: https://qualishealth.org/sites/default/files/White-Paper-Oral-Health-Primary-Care.pdf. Accessed April 30, 2021.

3. Casciaro T, Edmondson A, Jan S. Cross-silo leadership. Harv Bus Rev. 2019;97:130-139.

4. Ellner AL, Phillips RS. The coming primary care revolution. J Gen Intern Med. 2017;32(4):381-386. doi:10.1007/s11606-016-3944-3

5. Institute of Medicine (IOM). Dental Education at the Crossroads: Challenges and Change. Washington, DC: The National Academies Press; 1995.

6. US Department of Health and Human Services. Oral health in America: a report of the surgeon general. Rockville, MD: US Department of Health and Human Services, National Institute of Dental and Craniofacial Research, National Institutes of Health; 2000.

7. Bailet HL, Formicola AJ. Introduction to "advancing dental education in the 21st century" project. J Dent Educ. 2017;81(8):1004-1007. doi:10.21815/JDE.017.052

8. Gies WJ. Dental education in the United States and Canada: a report to the Carnegie Foundation for the advancement of teaching. J AM Coll Dent. 2012;79(2):32-49.

9. Kleinman DV, Abel SN, Mosc NG. Help wanted: dental expertise to guide oral health integration into health care. J Am Dent Assoc. 2018;149 (6):404-406. doi:10.1016/j.adaj.2018.03.031

10. Menand L. The Marketplace of Ideas. New York, NY: W.W. Norton and Company; 2010.

11. Kamm AM, Liu JB, Demitroulas K, et al. An interdisciplinary approach to safe opioid prescribing and administration for surgical patients at an academic medical center. J Clin Nurs. 2020. doi:10.1111/jocn.15561

\section{Epilogue}

Steven J Scrivani

The world is a changing place, healthcare has a different model, societal concerns for good health and well-being have taken center stage and the need for improved pain management is ubiquitous and particularly for Dental Medicine. These Proceedings from this conference highlight some of the many issues that come to the forefront of the Dental profession and healthcare in general with regard to providing new, improved and individualized pain management for society.

\section{Acknowledgments}

This program was supported by grants from Coverys Community Healthcare Foundation and RIZE Massachusetts. We would like to thank the Office of Continuing Education at Tufts University School of Dental Medicine for organizing, promoting and presenting this continuing education conference.

\section{Disclosure}

Dr. Alexandre F. DaSilva co-created GeoPain and CLARAi, described in the current study for measuring pain, and is the co-founder and co-owner of the start-up MoxyTech, Inc. (Ann Arbor, MI) that licenses the GeoPain technology from the University of Michigan. He also reports grants from National Institute of Health (NIA: R35AG072262 NCCIH: R01AT010060 NINDS: R01NS094413 NIDCR: U01DE025633). Dr. Michael E Schatman is research consultant for Firstox and Modoscript, outside the submitted work. The authors report no other conflicts of interest in this work.

\section{Publish your work in this journal}

The Journal of Pain Research is an international, peer reviewed, open access, online journal that welcomes laboratory and clinical findings in the fields of pain research and the prevention and management of pain. Original research, reviews, symposium reports, hypothesis formation and commentaries are all considered for publication. The manuscript

Submit your manuscript here: https://www.dovepress.com/journal-of-pain-research-journal management system is completely online and includes a very quick and fair peer-review system, which is all easy to use. Visit http:// www.dovepress.com/testimonials.php to read real quotes from published authors. 